\section{(6) OPEN ACCESS}

\title{
Comprehensive genomic analyses associate UGT8 variants with musical ability in a Mongolian population
}

\author{
Hansoo Park, ${ }^{1,2}$ Seungbok Lee, ${ }^{1,3}$ Hyun-Jin Kim, ${ }^{1,3}$ Young Seok Ju, ${ }^{1,4}$ \\ Jong-Yeon Shin, ${ }^{1,5}$ Dongwan Hong, ${ }^{1,6}$ Marcin von Grotthuss, ${ }^{2}$ Dong-Sung Lee, ${ }^{1,3}$ \\ Changho Park, Jennifer Hayeon Kim, ${ }^{1}$ Boram Kim, ${ }^{7}$ Yun Joo Yoo, ${ }^{8}$ Sung-ll Cho, ${ }^{9}$ \\ Joohon Sung, ${ }^{9}$ Charles Lee, ${ }^{2}$ Jong-\|l Kim, ${ }^{1,3,5,7}$ Jeong-Sun Seo ${ }^{1,3,4,5,7}$
}

\begin{abstract}
- Additional data are published online only. To view these files please visit the journal online (http://dx.doi.org/10.1136/ jmedgenet-2012-101209).

${ }^{1}$ Medical Research Center, Genomic Medicine Institute (GMI), Seoul National University, Seoul, Korea ${ }^{2}$ Department of Pathology, Brigham and Women's Hospital and Harvard Medical School, Boston, Massachusetts, USA ${ }^{3}$ Department of Biomedical Sciences, Seoul National University Graduate School, Seoul, Korea

${ }^{4}$ Macrogen Inc., Seoul, Korea

${ }^{5}$ Psoma Therapeutics Inc., Seoul, Korea

${ }^{6}$ Division of Convergence Technology, Functional Genomics Branch, National Cancer Center, Goyang, Korea ${ }^{7}$ Department of Biochemistry and Molecular Biology, Seoul National University College of Medicine, Seoul, Korea ${ }^{8}$ Department of Mathematics Education, Seoul National University, Seoul, Korea ${ }^{9}$ Seoul National University School of Public Health, Seoul, Korea
\end{abstract}

\section{Correspondence to Dr Jeong-Sun Seo and Dr Jong-II Kim, Genomic Medicine Institute, Medical Research Center, Seoul National University College of Medicine 101 Daehak-ro, Jongno-gu, Seoul 110-799, Korea; jeongsun@snu.ac.kr, jongil@snu.ac.kr}

HP, SL and H-JK contributed equally

Received 4 August 2012 Revised 25 September 2012 Accepted 10 October 2012 Published Online First 1 November 2012

\section{ABSTRACT}

Background Musical abilities such as recognising music and singing performance serve as means for communication and are instruments in sexual selection. Specific regions of the brain have been found to be activated by musical stimuli, but these have rarely been extended to the discovery of genes and molecules associated with musical ability.

Methods A total of 1008 individuals from 73 families were enrolled and a pitch-production accuracy test was applied to determine musical ability. To identify genetic loci and variants that contribute to musical ability, we conducted family-based linkage and association analyses, and incorporated the results with data from exome sequencing and array comparative genomic hybridisation analyses.

Results We found significant evidence of linkage at 4q23 with the nearest marker D4S2986 (LOD=3.1), whose supporting interval overlaps a previous study in Finnish families, and identified an intergenic single nucleotide polymorphism (SNP) (rs1251078, $p=8.4 \times 10^{-17}$ ) near UGT8, a gene highly expressed in the central nervous system and known to act in brain organisation. In addition, a non-synonymous SNP in UGT8 was revealed to be highly associated with musical ability (rs4148254, $p=8.0 \times 10^{-17}$ ), and a $6.2 \mathrm{~kb}$ copy number loss near UGT8 showed a plausible association with musical ability $\left(p=2.9 \times 10^{-6}\right)$.

Conclusions This study provides new insight into the genetics of musical ability, exemplifying a methodology to assign functional significance to synonymous and noncoding alleles by integrating multiple experimental methods.

\section{INTRODUCTION}

Song as a communication signal and as an instrument in sexual selection has been recognised since it was first proposed by Darwin. ${ }^{1-3}$ Musical ability is a non-verbal and complex cognitive skill, and appears to have a latent biological basis in that infants can differentiate frequencies and 'carry a tune' without receiving extensive formal musical training.

Researchers have described certain aspects of how the architecture of the brain affects facets of musical ability. Perception and vocal production of singing seem to be based on the auditory and motor domains of the brain. ${ }^{4}$ Studies of impaired language skills with spared musical abilities and impaired musical abilities with normal language skills have revealed a dissociation between these two skill sets, ${ }^{6}$ leading to the proposal of a distinct mental module associated with separate neural substrates and a set of neurally isolatable processing components. A minority of humans exhibit extreme musical abilities in the form of either absolute pitch (the ability to accurately label tones with specific musical notes) or amusia (the inability to accurately identify and mimic tones). ${ }^{7} 8$

Recent studies have identified genetic components of musical ability. For example, absolute pitch has a significant familial basis and is predominant in females. ${ }^{9}$ A twin study has shown substantial heritability for musical ability ${ }^{10}$ and linkage studies have found loci for musical aptitude and absolute pitch. ${ }^{11} 12$ Some polymorphisms of specific genes in association with musical ability have begun to be reported, including variants of AVPR1A and SLC6A4. ${ }^{13} 14$

As part of the GENDISCAN study (GENe DIScovery for Complex traits in large isolated families of Asians of the Northeast), which was designed to investigate genetic influences on complex traits in extended Asian families of rural Mongolia, we investigated the processing of pitch using 1008 subjects from 73 families. It was expected that several points of the GENDISCAN study would increase the power of genetic loci discovery in normal complex traits, considering (1) the study population has little ethnic admixture, (2) consists of large extended families, and (3) represents a community-based population unbiased by health status. ${ }^{15}$

To overcome the difficulties of identifying genetic variations underlying common complex diseases, an approach that allows for recruitment of homogeneous and isolated populations was proposed. However, only a few studies have incorporated this approach due to difficulties in sample recruitments. The inner Mongolian steppes are still inhabited by small populations; geographically isolated populations are commonly found in rural provinces of Mongolia. We recruited Mongolian individuals from an isolated population with large extended pedigrees. These individuals possess a 
homogeneous genetic background and close genetic affinity to populations of the northern part of East Asia. ${ }^{16-19}$

Previously, binary familiarity tests have mostly been used to indicate whether or not each song part sounds similar to assess musical ability. ${ }^{10} 20-22$ By shifting the pitch of melody one semitone higher or lower, participants were asked to classify two melodies as the same or different. In this study, we created a test to analyse subjects' acoustic outputs followed by hearing specific tones using cochlear implants (CI). ${ }^{23} 24$ There are advantages to this approach, which include the possibility to study musical ability as a whole and the better availability of subjects. We determined the pitch discrimination limen with a simulated CI coding strategy and employed the complementary nature of linkage- and association-based methods for musical ability. The functional importance of results was screened through the incorporation of data from exome sequencing and array-based comparative genomic hybridisation (aCGH). This combined approach provides a method by which to discover additional novel genetic loci underlying complex traits.

\section{METHODS}

\section{Study subjects and phenotype measurement}

In 2006, a total of 2008 volunteers were recruited in Dashbalbar, Dornod Province, Mongolia for the GENDISCAN project, ${ }^{25-28}$ which was designed to discover the genetic backgrounds of several complex traits (figure 1). For this project, we selected an isolated population composed of large extended families. This population is highly appropriate for gene mapping research due to its genetic homogeneity, decreased environmental heterogeneity, and restricted geographical distribution. ${ }^{29}$ Extended multi-generation families comprising a small number of founders are known to increase the genetic power. ${ }^{30}$ Traits included in this project are summarised in online supplementary table S1.

In this study, we chose 1008 individuals who are derived from 73 extended families and have precise pedigree structures. Table 1 lists descriptive characteristics of the study population. The average age of the participants is 31.0 years and $51.6 \%$ are women. The family structure in this population is very complicated, with multiple generations and many family pairs such as 1794 parent-offspring pairs, 734 full-siblings, 395 half-siblings, and 888 avuncular pairs. The average family size and standard deviation are 19.6 and 11.3, respectively. Peripheral blood sample was collected for each study subject, and DNA was extracted according to standard protocols. The extracted DNA was stored in solution at $-20^{\circ} \mathrm{C}$.

To examine the musical ability of subjects, we used a pitchproduction accuracy (PPA) test based on the difference limen of a pitch paradigm in a psychophysical experiment with a simulated CI coding strategy. ${ }^{31}$ PPA is given by $\left(100-10 \times\left(\left|v_{i}-v_{s}\right| /\right.\right.$ $\left.v_{s} \times 100\right)$ ), subtracting 10 points for each $1 \%$ error, where $v_{s}$ is the standard auditory frequency emitted by a pitch-producing device and $v_{i}$ is the vocal pitch frequency produced by the individuals, who hear a specific tone through a headset and recite the sound. ${ }^{32}$ A harmonic tone complex with a sound pressure level of $70 \mathrm{~dB}$ intensity and sex-dependent fundamental frequency was used as a stimulus (see online supplementary table S2).

The participants with PPA values higher than 60 were categorised as individuals with good musical ability because they were consistently and accurately able to produce tones differing by less than a semitone from one another; the number of subjects with a PPA score over 60 was 357 (35.4\%). However, for further analyses, participants with borderline PPA values between 50 and 70 were excluded to eliminate ambiguous PPA values; the number of subjects with PPA score over 70 was 268 (31.1\%).

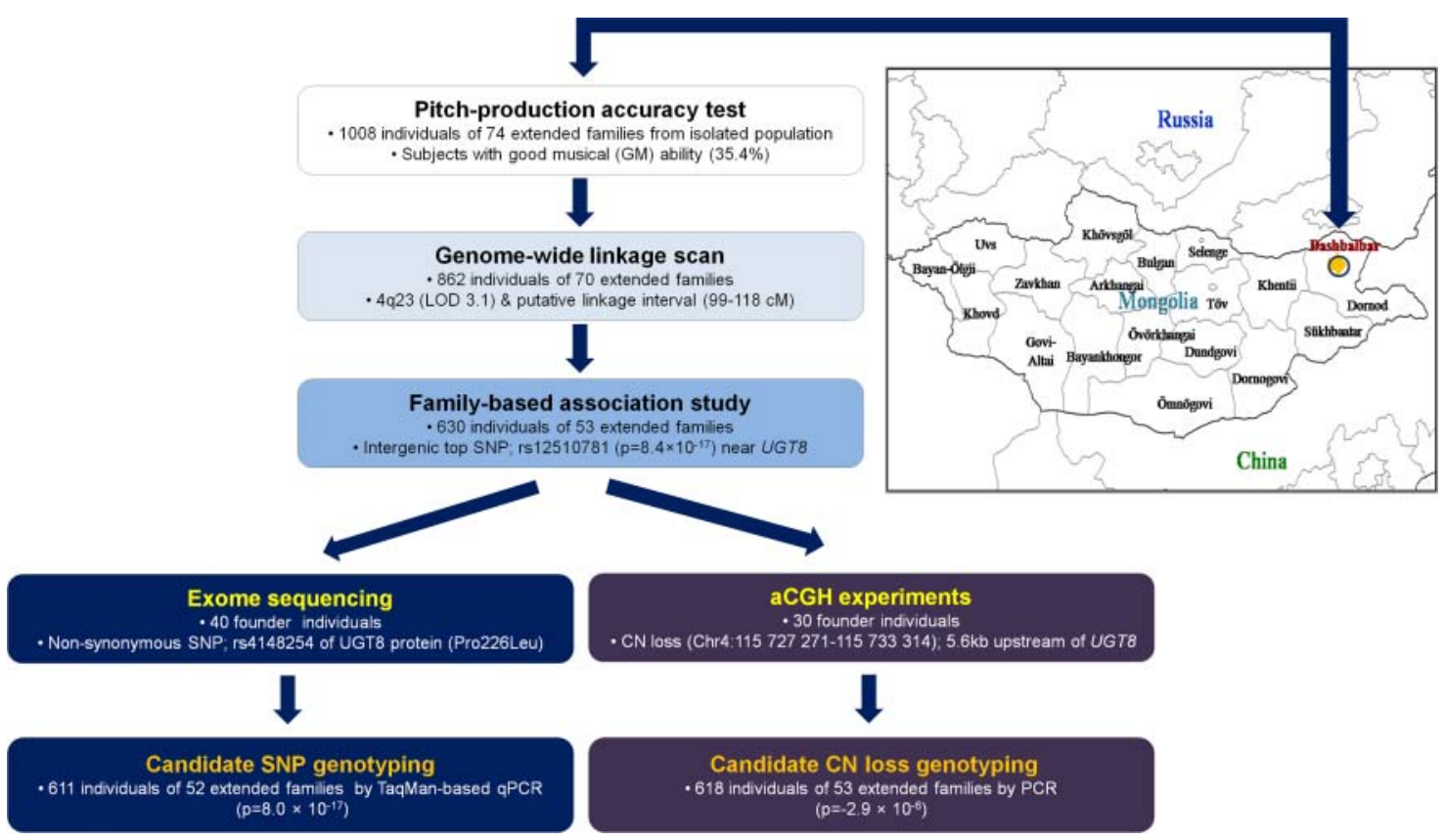

Figure 1 Overview of the project for musical ability. The pitch-production accuracy test was used to measure musical ability of 1008 individuals from 73 extended families of an isolated Mongolian population. We started with a genome-wide linkage study to identify potential causal loci associated with musical ability, and subsequently conducted a family-based association test under the linkage peak on 4q23 (99-118 cM). Furthermore, we used exome sequencing data in 40 founders and assessed copy number variants in 30 founders to explore plausible candidates for causal variants of musical ability with additional validating experiments. CN, copy number; SNP, single nucleotide polymorphism. 
Table 1 Descriptive characteristics of study participants

\begin{tabular}{ll}
\hline Characteristics & Value \\
\hline Sample information & \\
No. of samples & 1008 \\
No. of females (\%) & $520(51.6)$ \\
Mean (SD) age (in years) & $31.0(15.5)$ \\
No. of sample with PPA score (\%) & \\
$\geq 70$ & $268(26.6)$ \\
$\geq 60$ & $357(35.4)$ \\
$<60$ & $651(64.6)$ \\
$<50$ & $594(58.9)$ \\
Family information & \\
No. of families & 73 \\
Mean size (SD) of family members & $19.61(11.3)$ \\
No. of pairs & \\
Parent-offspring & 1794 \\
Full-sibling & 734 \\
Sister-sister & 198 \\
Brother-brother & 167 \\
Sister-brother & 369 \\
Half-sibling & 395 \\
Grandparent-grandchild & 1202 \\
Avuncular pairs & 888 \\
First cousins & 598 \\
\hline
\end{tabular}

PPA, pitch-production accuracy.

\section{Genome-wide linkage scan and family-based association study under linkage region}

We genotyped 862 samples from 70 families with deCODE 1039 microsatellite marker platform throughout the autosomes for genome-wide linkage analysis. We checked family relationships through PREST ${ }^{33}$ using an average identity-by-descent (IBD)-based method. PEDCHECK was used to examine Mendelian inconsistencies in genotype data, ${ }^{34}$ and nonMendelian genotype errors were detected with SimWalk. ${ }^{35}$ After fixing the genotype errors, multipoint identity-bydescent-matrices were calculated at each $1 \mathrm{cM}$ distance, and converted using the Markov chain-Monte Carlo method by LOKI. ${ }^{36}$ We used the Kosambi mapping function (derived from the deCODE map) to convert map distances into recombination fractions. For the multipoint linkage analyses, the Sequential Oligogenic Linkage Analysis Routines package was used. ${ }^{37}$ We performed 10000 permutation tests using the lodadj option to obtain the empirical $\mathrm{p}$ value. In addition, we estimated the adjusted narrow-sense heritability $\left(h^{2}\right)$ (ie, the proportion of phenotype variance attributable to additive genetic variance). In all analyses, we used age and sex as covariates

For further association analysis, 53 extended families composed of 630 family members were genotyped using an Illumina Human610-Quad BeadChip kit by Macrogen (Macrogen Inc, Seoul, Korea). We evaluated the Mendelian inconsistencies in single nucleotide polymorphism (SNP) data using PEDCHECK. ${ }^{34}$ Non-Mendelian genotype errors were detected using Merlin. ${ }^{38}$ SNP quality control assessment was based on SNP call rate, marker error rate, and minor allele frequency (MAF); minimum per-SNP call rate of $99 \%$, less than $1 \%$ marker error rate, and higher than 5\% MAF. In addition, we also removed genotypes with Hardy-Weinberg equilibrium $\mathrm{p}$ values $<1 \times 10^{-6}$. We focused on the putative linkage region in chromosome 4 for this analysis (1-LOD Unit Support Interval: $99-118 \mathrm{cM}$ ). A total of 3424 SNPs that met quality control criteria were included in the putative linkage region, and the PBAT tool in HelixTree software (V.6.4; GoldenHelix) was used for family-based association test (FBAT), which can control population stratification or population admixture. ${ }^{15} 39$ The null hypothesis was 'linkage and no association (sandwich variance), 40 which can be useful for expanded pedigrees by calculating a robust variance. We used the generalised estimating equation for the FBAT test statistic, and hypothesised an additive model. The association result was adjusted by covariates of age and sex.

\section{Screening functional significance of candidates using exome sequencing and aCGH data integration}

To assign a functional significance to candidates, we used exome sequencing data of 40 founders and 180K aCGH results of 30 founders, both of which were included in this study and previously genotyped in our group. The experimental summary of each is described in data supplement (see online supplementary tables S3-S5, supplementary methods). Among SNPs and short insertions/deletions (indels) called from exomes, we selected coding sequence SNPs and indels, and canonical splicesite variants as candidates, along with the copy number variants (CNVs) called from the aCGH experiment. Focusing on variants in the putative linkage region, we further narrowed our candidates by linkage disequilibrium (LD) estimation with the top 10 SNPs of our association study. Haploview software (V.3.2) was used for this LD estimation.

Among the candidates showing a significant level of LD, we selected one SNP and one CNV to be genotyped in our study population and compared their $\mathrm{p}$ values with the association results. For the SNP selected, three-dimensional (3D) modelling was conducted to predict its functional impact on the corresponding protein (see online supplementary methods).

\section{RESULTS}

\section{Family-based linkage and association study}

The heritability explained by the additive genetic portion of musical ability was estimated as $40 \%(\mathrm{p}<0.0001,95 \%$ CI $20.4 \%$ to $59.6 \%$ ), and linkage regions with LOD $>1.0$ were found for musical ability from the genome-wide linkage scan (see online supplementary table S6). The maximum LOD score was 3.1 at chromosome 4q23 with the nearest marker D4S2986 (figure $2 \mathrm{~A}$ ), and the putative linkage region encompassing a maximum 1-LOD unit supports an interval range from $99 \mathrm{cM}$ to $118 \mathrm{cM}$ (figure $2 \mathrm{~B}$ ). In the next phase, we conducted FBAT to identify candidate variants within the putative linkage interval. Table 2 shows the top 10 SNPs that were significantly associated with musical ability, and all of these have reached the strict genome-wide significance of $p<1 \times 10^{-8}$. The strongest association $\left(p=8.4 \times 10^{-17}\right)$ was found for rs12510781, an intergenic SNP near UGT8 (MIM 601291). The regional association plot near UGT8 is shown in figure 2C, and plotted recombination rates reflecting local $\mathrm{LD}$ structure were estimated from HapMap data. Three other SNPs (rs10024217, rs1903364, and rs12504058) were in moderate LD with $\mathrm{rs} 12510781\left(\mathrm{r}^{2}=0.4\right)$. A synonymous SNP within UGT8 (rs4148255) also showed significance in $\mathrm{p}$ value levels, despite the low LD with rs12510781 $\left(p=2.7 \times 10^{-10}, r^{2}<0.1\right)$. The SNP with the second highest significance $\left(p=3.0 \times 10^{-13}\right)$ was $\mathrm{rs} 9307160$ in the intron of UNC5C (MIM 603610), and the others were located near ALPK1 (MIM 607347) and ELOVL6 (MIM 611546). 
A

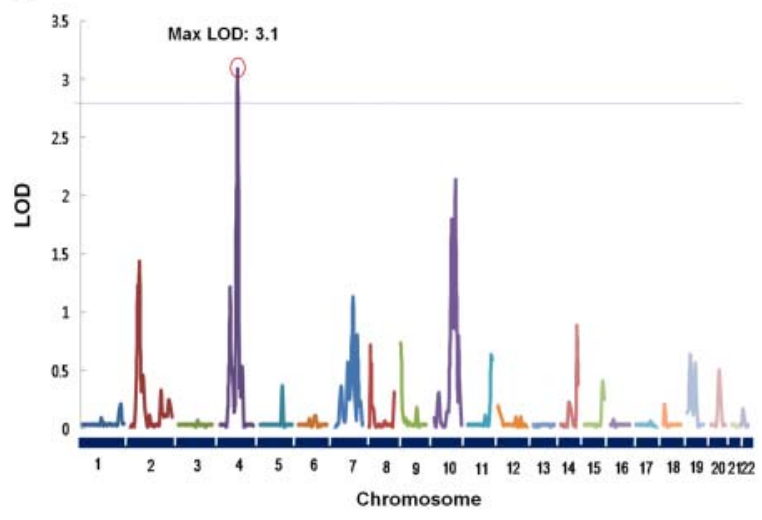

C

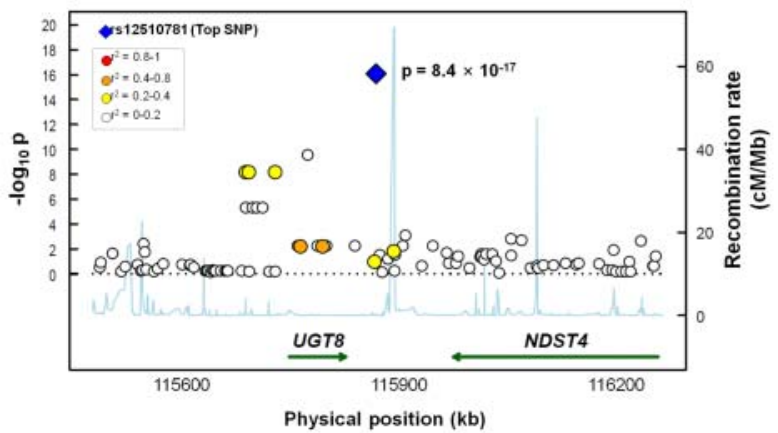

B
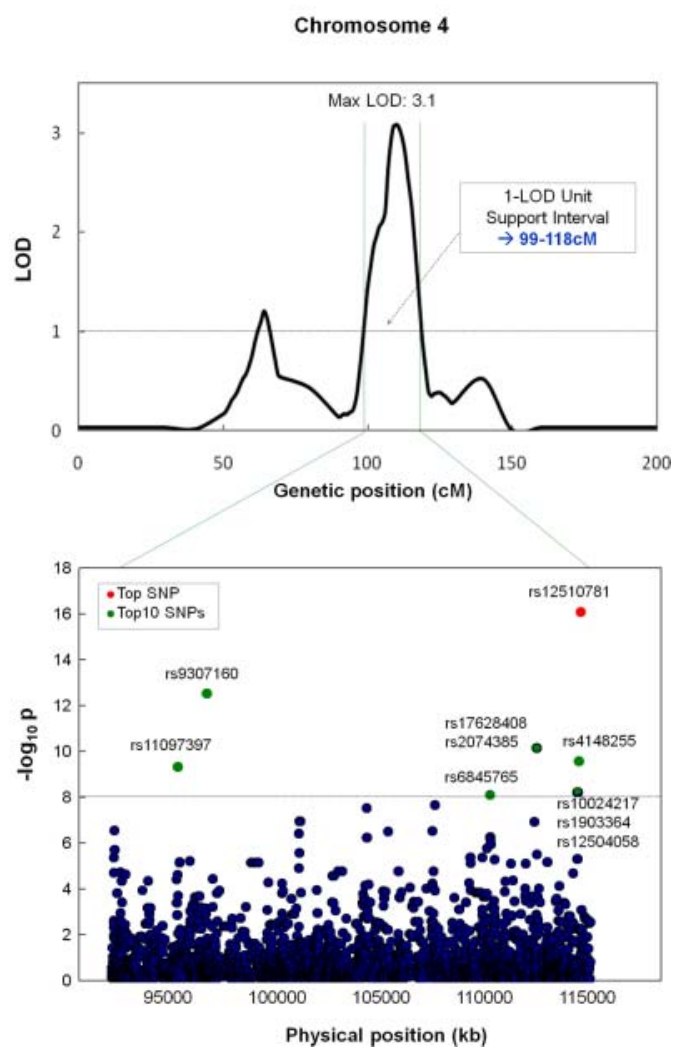

Figure 2 Summary of genome-wide linkage and association results for musical ability. (A) Genome-wide linkage results for musical ability. (B) The linkage peak on chromosome 4 and association plot under the linkage support region. The linkage support interval is indicated by a green line (99-118 cM). The red dot is the top single nucleotide polymorphism (SNP) by family-based association test. The SNPs 2-10 are labelled with green dots. (C) Regional plot of association results for SNPs from analysis $\left(-\log _{10} \mathrm{p}\right)$ for UGT8 $( \pm 300 \mathrm{~kb}$ position from top SNP). The SNPs close to rs12510781, the most significant SNP (blue diamond), are colour-coded to reflect their linkage disequilibrium with this $\operatorname{SNP}\left(\mathrm{r}^{2}<0.2\right.$; white, $0.2 \leq r^{2}<0.4$; yellow, $0.4 \leq r^{2}<0.8$; orange, $r^{2} \geq 0.8$; red).

\section{Utilisation of exome sequencing and aCGH data to assign functional significance to candidate variants}

Among the candidates from the exome data (347 SNPs and seven indels in the putative linkage region), we narrowed down to four SNPs that were in strong LD with the top 10 SNPs identified via FBAT $\left(r^{2}>0.6\right.$, online supplementary table S7). We found that a non-synonymous SNP (nsSNP) in UGT8 (rs4148254) showed perfect LD with rs12510781, the most significant SNP from FBAT $\left(r^{2}=1.0\right)$, and this SNP was genotyped in 611 FBAT samples for the association analysis. As a result, the $\mathrm{LD}$ between rs4148254 and rs12510781 was re-estimated $\left(\mathrm{r}^{2}=0.93\right)$, and the rs4148254 SNP was found to have the most significant association with musical ability in this study $\left(p=8.0 \times 10^{-17}\right)$. The effect estimate of this SNP in founder samples was also higher than that of $\mathrm{rs} 12510781(\mathrm{OR}=3.4$, $95 \%$ CI 1.2 to 9.9 vs OR=3.0, 95\% CI 1.1 to 8.2 , online supplementary tables $\mathrm{S} 8, \mathrm{~S} 9)$. The 3D modelling of UGT8 protein showed that Pro226, which is changed to leucine by the SNP,

Table 2 Top 10 SNPs significantly associated with musical ability by FBAT under the putative linkage region of chromosome 4

\begin{tabular}{|c|c|c|c|c|c|c|c|}
\hline \multirow[b]{2}{*}{ SNP } & \multirow[b]{2}{*}{ *Position } & \multicolumn{2}{|l|}{ Alleles } & \multirow{2}{*}{$\begin{array}{l}\text { Frequency of } \\
\text { effect allele }\end{array}$} & \multirow[b]{2}{*}{ p Value (FBAT) } & \multirow[b]{2}{*}{ tNearest gene(s) } & \multirow[b]{2}{*}{ Location (distance) } \\
\hline & & Effect & Other & & & & \\
\hline rs12510781 & 115860030 & G & $\mathrm{A}$ & 0.12 & $8.4 \times 10^{-17}$ & UGT8 & Intergenic (42.3 kb) \\
\hline rs 9307160 & 96586977 & C & $\mathrm{T}$ & 0.10 & $3.0 \times 10^{-13}$ & UNC5C & Intronic $\left(^{-}\right)$ \\
\hline rs2074385 & 113598098 & C & A & 0.91 & $7.1 \times 10^{-11}$ & ALPK1 & Intergenic (14.8 kb) \\
\hline rs4148255 & 115764226 & A & G & 0.88 & $2.7 \times 10^{-10}$ & UGT8 & Synonymous $\left(^{-}\right)$ \\
\hline rs11097397 & 95087875 & $G$ & $\mathrm{~T}$ & 0.28 & $4.8 \times 10^{-10}$ & - & Intergenic $\left(^{-}\right)$ \\
\hline rs 12504058 & 115718566 & G & A & 0.28 & $6.1 \times 10^{-9}$ & UGT8 & Intergenic $(20.4 \mathrm{~kb})$ \\
\hline rs6845765 & 111177613 & $\mathrm{C}$ & $\mathrm{T}$ & 0.86 & $8.2 \times 10^{-9}$ & ELOVL6 & Intergenic (12.0 kb) \\
\hline
\end{tabular}

*Positions are based on Build 36 from NCBI.

tNearest gene, within $\pm 100 \mathrm{~kb}$ of the SNP.

FBAT, family-based association test; SNP, single nucleotide polymorphism 
might be part of the loop exposed outside of the predicted 3D structure, and the loop with the Pro226 residue contains sequence motifs including TRFH domain docking and USP7-binding motifs (see online supplementary figure S1).

At the level of CNVs, only one copy number (CN) loss was found to have moderate LD with rs4148255, the fifth most significant SNP in FBAT $\left(\mathrm{r}^{2}=0.48\right.$; online supplementary table S10). This CN loss (Chr4: 115727 257-115 733 452) is located $5.6 \mathrm{~kb}$ upstream of the UGT8 gene. We genotyped it in 618 FBAT samples and the frequencies of heterozygous and homozygous $\mathrm{CN}$ losses were shown to be $45.15 \%$ and $10.03 \%$ in our study subjects (allele frequency $=32.61 \%$ ). This CNV was negatively associated with musical ability $\left(p=2.9 \times 10^{-6}\right)$ and, interestingly, a diploid status at this position was shown to potentiate the positive effect of rs4148254 in founders (see online supplementary table S11). In addition, we identified a significant interaction effect between this CNV and rs4148254 using a logistic regression model $(p=0.01)$.

\section{DISCUSSION}

In this study, we explored the genetic determinants of musical ability by combining several methodologies, namely familybased linkage and association studies supported by exome sequencing and aCGH data analyses. This study was conducted as a part of the GENDISCAN project, which was designed to discover the genetic backgrounds of complex traits in Mongolia.

Musical ability is a well-known complex trait determined by multiple environmental and genetic factors. As this trait consists of several factors including perception, cognition, learning, and emotions, a variety of genes have an effect on one's musical ability, both independently and interactively. To discover genetic backgrounds of these complex traits, studies should be designed from the first to increase the power to detect genetic loci. In this regard, our study has some strong points as described in the Introduction and Methods, which include little ethnic admixture and large extended families. In addition, we excluded samples with borderline phenotypes from all the analyses to derive more accurate results.

Our results support the view that musical ability is heritable and have shown significant evidence of linkage for musical ability in large families. Previously, a linkage study for musical aptitude was performed with samples in a small number of Finnish multigenerational families, composed of predominantly white subjects. That study found an association of the chromosomal region 4q22 with musical aptitude in the Finnish study population, ${ }^{11}$ which overlaps with our linkage interval on chromosome 4q. Despite several differences in methodology, we believe that overlapping results for musical ability in different ethnic populations enhance the reliability of this linkage region on chromosome 4q.

We also discovered common variants strongly associated with musical ability, suggesting a biological mechanism for this finding. Including the most significant, five SNPs among the top 10 were shown to lie near or within UGT8. In addition, there was no LD structure between rs12510781 and rs4148255. These two unrelated variants on one gene, associated with the same phenotype, increase the possibility of UGT8 being one of the true susceptibility genes for musical ability.

To identify more detailed causal variants, we integrated additional technologies such as exome sequencing and aCGH, resulting in the discovery of another nsSNP in UGT8 and a CN loss located $5.6 \mathrm{~kb}$ upstream of this gene. The SNP rs4148254, which changes amino acid 226 of the UGT8 protein from proline to leucine, was not included in the platform we used, and has shown a lower $p$ value than rs12510781 in our study population (see online supplementary figure S1A,B). Because the BLOSUM score ${ }^{41}$ for this change is ' -3 ', and PolyPhen- $2^{42}$ predicts this to be damaging, the SNP might affect the function of the UGT8 protein. Moreover, this proline amino acid seems to be conserved among vertebrates (see online supplementary table S12). The three other SNPs (rs35308602, rs2074381, and rs3828539), which were in high LD $\left(r^{2}>0.6\right)$ with the top 10 SNPs, were predicted to be benign by PolyPhen- 2 and the BLOSUM scores were '2', ' 1 ', and ' -1 ', respectively (see online supplementary table S7). In case of the CN loss, even though it was not more significant than the associated SNP allele, the synergetic effect of this variant with rs4148254 was suggested in the founder analysis.

The protein encoded by UGT8 is UDP glycosyltransferase 8, which is highly expressed in brain (see online supplementary figure S2). It is the first enzyme involved in complex lipid biosynthesis in the myelinating oligodendrocyte ${ }^{43}$ and clearance of long-chain ceramides (lcCer). lcCer clearance in neurons is mediated by glucosylceramide synthase (GCS) and studies have shown that decreased GCS leads to abnormally high lcCer. ${ }^{44}$ A significant early downregulation in glial GCS expression was associated with an increase in UGT8 mRNA in Alzheimer's disease, ${ }^{45}$ and some patients with Alzheimer's disease have been observed to preserve musical ability long after losing all other cognitive functions. ${ }^{6}$

Although this study primarily focused on UGT8, there are other genes such as UNC5C, ALPK1, and ELOVL6 equally worth our attention. The protein encoded by UNC5C plays a role in the chemorepulsive effect of netrin-1 in axon guidance. This gene was previously suggested as a susceptibility gene for musical ability in the Finnish linkage study. ${ }^{11}$ Regarding the other two, one study has shown that mice homozygous for disrupted copies of Alpk1 exhibited coordination defects, ${ }^{46}$ and ELOVL6 was once reported as one of the susceptibility loci for attention-deficit/hyperactivity disorder in a genome-wide association study. ${ }^{47}$ Several previous findings, as listed above, have supported the neural involvement of those candidate genes; however, more evidence should be given to associate them with musical ability.

Music is a complex cognitive skill in the neuronal network affected by several potential covariates. We first considered language ability as a potential covariate besides age and sex. However, we found no language skill defects in our study subjects, and previous studies have reported that it is possible for language skills to be impaired while musical abilities are spared (aphasia without amusia); likewise, musical abilities can be impaired while language skills are spared (amusia without aphasia). ${ }^{6} 48$ In addition, more factors including special musical training, education status, and education duration might be considered as potential covariates, since it has been reported that the skill of absolute pitch could be developed at a very young age by special musical training. ${ }^{49} 50$ However, our participants lived in an isolated area with a homogeneous culture, and most of them were educated in the same public school without any additional musical training. In this study, therefore, we did not take those factors into account for analyses.

In summary, we have demonstrated for the first time that common genetic variants in UGT8 are associated with musical ability, exemplifying a methodology to assign functional significance to the results of various association studies, which in many cases yield synonymous or non-coding alleles. 
Acknowledgements The authors appreciate the help of all study participants and collaborators. We thank Omer Gokcumen and Raju Govindaraju at Harvard Medical School and Thomas Bleazard at Seoul National University for their personal comments regarding this manuscript.

Contributors J-SS planned and managed the project. J-IK, HP, YSJ, S-IC, and JS recruited and measured phenotypes of the Mongolian samples. HP, H-JK, YJY and J-IK analysed linkage data and family-based association studies. SL, J-YS, DH, and J-IK executed exome sequencing and analysed sequence data. HP, SL, D-SL, CP, JHK, and BK executed and analysed aCGH experiments. MvG performed the 3D modelling and motif analysis. CL supervised research at Brigham and Women's Hospital/Harvard Medical School. J-SS, HP, H-JK, SL, and J-IK wrote the manuscript and CL edited the manuscript.

Funding This work was supported by the Korean Ministry of Education, Science and Technology (Grant No. 2003-2001558) and the US National Institutes of Health (Grant No. HG004221)

\section{Competing interests None.}

Ethics approval This study was approved by the Institutional Review Board of the Seoul National University Hospital (approval number, H-0307-105-002).

Data sharing statement The whole data of exome and aCGH experiments, some of which were used for this study, have not been published yet. We can provide the part of data related to this project upon request.

\section{REFERENCES}

1. Darwin C. The descent of man, and selection in relation to sex. New York: D. Appleton and company, 1871

2. Miller GF. The mating mind: how sexual choice shaped the evolution of human nature. 1st edn. New York: Doubleday, 2000.

3. Fitch WT. The biology and evolution of music: a comparative perspective. Cognition 2006;100:173-215

4. Peretz I, Coltheart M. Modularity of music processing. Nat Neurosci 2003;6:688-91.

5. Coltheart M. Modularity and cognition. Trends Cogn Sci 1999;3:115-20.

6. Halpern AR, O'Connor MG. Implicit memory for music in Alzheimer's disease. Neuropsychology 2000;14:391-7.

7. Peretz I, Cummings S, Dube MP. The genetics of congenital amusia (tone deafness): a family-aggregation study. Am J Hum Genet 2007;81:582-8.

8. Peretz I, Ayotte J, Zatorre RJ, Mehler J, Ahad P, Penhune VB, Jutras B. Congenital amusia: a disorder of fine-grained pitch discrimination. Neuron 2002;33:185-91.

9. Profita J, Bidder TG. Perfect pitch. Am J Med Genet 1988;29:763-71.

10. Drayna D, Manichaikul A, de Lange M, Snieder H, Spector T. Genetic correlates of musical pitch recognition in humans. Science 2001;291:1969-72.

11. Pulli K, Karma K, Norio R, Sistonen P, Goring HH, Jarvela I. Genome-wide linkage scan for loci of musical aptitude in Finnish families: evidence for a major locus at 4q22. J Med Genet 2008;45:451-6.

12. Theusch $\mathbf{E}$, Basu A, Gitschier J. Genome-wide study of families with absolute pitch reveals linkage to 8q24.21 and locus heterogeneity. Am J Hum Genet 2009;85:112-19.

13. Ukkola LT, Onkamo P, Raijas P, Karma K, Jarvela I. Musical aptitude is associated with AVPR1A-haplotypes. PLoS One 2009;4:e5534.

14. Morley AP, Narayanan M, Mines R, Molokhia A, Baxter S, Craig G, Lewis CM, Craig I. AVPR1A and SLC6A4 polymorphisms in choral singers and non-musicians: a gene association study. PLoS One 2012; 7:e31763

15. Ott J, Kamatani Y, Lathrop M. Family-based designs for genome-wide association studies. Nat Rev Genet 2011;12:465-74.

16. Katoh T, Mano S, Ikuta T, Munkhbat B, Tounai K, Ando H, Munkhtuvshin N, Imanishi $\mathrm{T}$, Inoko $\mathrm{H}$, Tamiya $\mathrm{G}$. Genetic isolates in East Asia: a study of linkage disequilibrium in the X chromosome. Am J Hum Genet 2002; 71:395-400.

17. Nakajima T, Wooding S, Sakagami T, Emi M, Tokunaga K, Tamiya G, Ishigami T, Umemura S, Munkhbat B, Jin F, Guan-Jun J, Hayasaka I, Ishida T, Saitou N, Pavelka $\mathrm{K}$, Lalouel JM, Jorde LB, Inoue I. Natural selection and population history in the human angiotensinogen gene (AGT): 736 complete AGT sequences in chromosomes from around the world. Am J Hum Genet 2004:74:898-916.

18. Katoh T, Munkhbat B, Tounai K, Mano S, Ando H, Oyungerel G, Chae GT, Han H, Jia GJ, Tokunaga K, Munkhtuvshin N, Tamiya G, Inoko H. Genetic features of Mongolian ethnic groups revealed by Y-chromosomal analysis. Gene 2005;346:63-70.

19. Kimura T, Kobayashi T, Munkhbat B, Oyungerel G, Bilegtsaikhan T, Anar D, Jambaldorj J, Munkhsaikhan S, Munkhtuvshin N, Hayashi H, Oka A, Inoue I, Inoko H. Genome-wide association analysis with selective genotyping identifies candidate loci for adult height at 8q21.13 and 15q22.33-q23 in Mongolians. Hum Genet 2008;123:655-60.

20. Kalmus H, Fry DB. On tune deafness (dysmelodia): frequency, development genetics and musical background. Ann Hum Genet 1980;43:369-82.
21. Peretz I, Gaudreau D, Bonnel AM. Exposure effects on music preference and recognition. Mem Cognit 1998;26:884-902

22. Ayotte J, Peretz I, Hyde K. Congenital amusia: a group study of adults afflicted with a music-specific disorder. Brain 2002;125(Pt 2):238-51.

23. Leal MC, Shin YJ, Laborde ML, Calmels MN, Verges S, Lugardon S, Andrieu S, Deguine 0 , Fraysse B. Music perception in adult cochlear implant recipients. Acta Otolaryngol 2003;123:826-35.

24. Haumann S, Mühler R, Ziese M, Specht H. Diskrimination musikalischer Tonhöhen bei Patienten mit Kochleaimplantat. HNO 2007;55:613-19.

25. Ju YS, Park H, Lee MK, Kim Jl, Sung J, Cho SI, Seo JS. A genome-wide Asian genetic map and ethnic comparison: the GENDISCAN study. BMC Genomics 2008;9:554.

26. Im SW, Kim HJ, Lee MK, Yi JH, Jargal G, Sung J, Cho SI, Kim Jl. Genome-wide linkage analysis for ocular and nasal anthropometric traits in a Mongolian population. Exp Mol Med 2010;42:799-804.

27. Paik SH, Kim HJ, Son HY, Lee S, Im SW, Ju YS, Yeon JH, Jo SJ, Eun HC, Seo JS, Kwon OS, Kim Jl. Gene mapping study for constitutive skin color in an isolated Mongolian population. Exp Mol Med 2012:44:241-9.

28. Lee MK, Cho SI, Kim H, Song YM, Lee K, Kim Jl, Kim DM, Chung TY, Kim YS Seo JS, Ham DI, Sung J. Epidemiologic characteristics of intraocular pressure in the Korean and Mongolian populations: the Healthy Twin and the GENDISCAN study. Ophthalmology 2012;119:450-7.

29. Kristiansson K, Naukkarinen J, Peltonen L. Isolated populations and complex disease gene identification. Genome Biol 2008;9:109.

30. Arcos-Burgos M, Muenke M. Genetics of population isolates. Clin Genet 2002;61:233-47.

31. Haumann S, Muhler R, Ziese M, von Specht H. (Discrimination of musical pitch with cochlear implants). HNO 2007:55:613-19.

32. Nikjeh DA, Lister JJ, Frisch SA. The relationship between pitch discrimination and vocal production: comparison of vocal and instrumental musicians. J Acoust Soc Am 2009;125:328-38.

33. McPeek MS, Sun L. Statistical tests for detection of misspecified relationships by use of genome-screen data. Am J Hum Genet 2000;66:1076-94.

34. O'Connell JR, Weeks DE. PedCheck: a program for identification of genotype incompatibilities in linkage analysis. Am J Hum Genet 1998;63:259-66.

35. Weeks DE, Sobel E, O'Connell JR, Lange K. Computer programs for multilocus haplotyping of general pedigrees. Am J Hum Genet 1995;56:1506-7.

36. Heath SC. Markov chain Monte Carlo segregation and linkage analysis for oligogenic models. Am J Hum Genet 1997;61:748-60.

37. Almasy L, Blangero J. Multipoint quantitative-trait linkage analysis in general pedigrees. Am J Hum Genet 1998;62:1198-211.

38. Abecasis GR, Cherny SS, Cookson WO, Cardon LR. Merlin-rapid analysis of dense genetic maps using sparse gene flow trees. Nat Genet 2002;30:97-101.

39. Lange C, DeMeo D, Silverman EK, Weiss ST, Laird NM. PBAT: tools for family-based association studies. Am J Hum Genet 2004;74:367-9.

40. Laird NM, Lange C. Family-based designs in the age of large-scale gene-association studies. Nat Rev Genet 2006;7:385-94.

41. Henikoff $\mathbf{S}$, Henikoff JG. Amino acid substitution matrices from protein blocks. Proc Natl Acad Sci U S A 1992;89:10915-19.

42. Adzhubei IA, Schmidt S, Peshkin L, Ramensky VE, Gerasimova A, Bork P, Kondrashov AS, Sunyaev SR. A method and server for predicting damaging missense mutations. Nat Methods 2010;7:248-9.

43. Dugas JC, Tai YC, Speed TP, Ngai J, Barres BA. Functional genomic analysis of oligodendrocyte differentiation. J Neurosci 2006;26:10967-83.

44. Han X, D MH, McKeel DW Jr, Kelley J, Morris JC. Substantial sulfatide deficiency and ceramide elevation in very early Alzheimer's disease: potential role in disease pathogenesis. J Neurochem 2002;82:809-18.

45. Marks N, Berg MJ, Saito M. Glucosylceramide synthase decrease in frontal cortex of Alzheimer brain correlates with abnormal increase in endogenous ceramides: consequences to morphology and viability on enzyme suppression in cultured primary neurons. Brain Res 2008;1191:136-47.

46. Chen M, Xu R. Motor coordination deficits in Alpk1 mutant mice with the inserted piggyBac transposon. BMC Neurosci 2011;12:1.

47. Mick E, Todorov A, Smalley S, Hu X, Loo S, Todd RD, Biederman J, Byrne D, Dechairo B, Guiney A, McCracken J, McGough J, Nelson SF, Reiersen AM, Wilens TE, Wozniak J, Neale BM, Faraone SV. Family-based genome-wide association scan of attention-deficit/hyperactivity disorder. J Am Acad Child Adolesc Psychiatry 2010;49:898-905 e3.

48. Barlett JC, Halpern AR, Dowling WJ. Recognition of familiar and unfamiliar melodies in normal aging and Alzheimer's disease. Mem Cognit 1995:23:531-46.

49. Takeuchi AH, Hulse SH. Absolute pitch. Psychol Bull 1993;113:345-61.

50. Gregersen PK, Kowalsky E, Kohn N, Marvin EW. Early childhood music education and predisposition to absolute pitch: teasing apart genes and environment. Am J Med Genet 2001:98:280-2. 